\title{
Optical Ozone Sensing by Composite Films of Noble Metals and CdSe/ZnS Quantum Dots
}

\author{
Masanori Ando ${ }^{1}$, Vasudevanpillai Biju ${ }^{2}$, Hideya Kawasaki ${ }^{3}$, Yasushi Shigeri ${ }^{4}$ \\ ${ }^{1}$ Biomedical Research Institute, National Institute of Advanced Science and Technology (AIST), \\ 1-8-31 Midorigaoka, Ikeda, Osaka 563-8577, Japan \\ ${ }^{2}$ Research Institute for Electronic Science, Hokkaido University, \\ N20W10, Kita-Ward, Sapporo, Hokkaido 001-0020, Japan \\ ${ }^{3}$ Faculty of Chemistry, Materials and Bioengineering, Kansai University, \\ 3-3-35 Yamate-cho, Suita, Osaka 564-8680, Japan \\ ${ }^{4}$ Health Research Institute, National Institute of Advanced Science and Technology (AIST), \\ 2217-14 Hayashi-cho, Takamatsu, Kagawa 761-0395, Japan \\ m-ando@aist.go.jp, yasushi.shigeri@aist.go.jp
}

\begin{abstract}
The photoluminescence-based ozone sensitivity of composite films composed of noble metal particles and $\mathrm{CdSe} / \mathrm{ZnS}$ core-shell quantum dot is investigated. The composite films are prepared by the sputter-deposition of $\mathrm{Pt}, \mathrm{Au}, \mathrm{Pt}-\mathrm{Pd}$ alloy or $\mathrm{Ag}$ particles onto a glass plate substrate, which is followed by deposition of quantum dots from an organic solution. For comparison, a quantum dot film without any noble metal is prepared. In air, the Pt-, Au- and Pt-Pd alloy-based composite films, and quantum dot-only film emit bright photoluminescence, whereas emission from Ag-based composite film is weak. The composite films and quantum dot-only film show reversible changes in photoluminescence intensity in presence of ozone at the ppm level in air at room temperature. Optical ozone sensitivity, i.e., ozone-induced changes in photoluminescence intensity of quantum dot, are affected by the noble metal particles in the film. When compared with a quantum dot-only film, a composite film composed of $\mathrm{Pt}$ or Au shows higher ozone sensitivity by $43 \%$ or $27 \%$, respectively. In contrast, the sensitivity of a composite film composed of Pt-Pd alloy particles is lower than that of a quantum dot-only film by $32 \%$. However, the alloy- and Au-based films show quicker recovery of photoluminescence, which is after removal of ozone in the surrounding atmosphere, when compared with quantum dot-only and Ptbased film. These results show a possibility to enhance the optical ozone sensitivity and response/recovery rate of quantum dots by the addition of noble metal particles.
\end{abstract}

Key words: optical ozone sensor, photoluminescence, quantum dot, noble metal, composite film

\section{Introduction}

Utilization of ozone gas is expanding as a strong and clean oxidizing agent for purification of air and water, manufacture of semiconductors, medicines, esthetics and chemicals. However, ozone is harmful to human health when its concentration exceeds a few ppm in air. Therefore, a requirement for the expedient sensing of ozone in air is growing.

Optical sensors have advantages over electronic sensors, such as resistance to electromagnetic noise, safety against fire caused by electric spark, remote/noncontact signal readout, and compatibility with optical fibers and waveguides. We have recently reported that thin films of CdSe-based coreshell quantum dots (QDs) show ozone-induced quenching of photoluminescence (PL) and reversible recovery of PL in pure air [1]. This phenomenon is expected to be used for ozone sensor having advantages over conventional ozone detection techniques [2]. We hypothesize that ozone-induced $\mathrm{PL}$ quenching originates from changes in the ratio of radiative to nonradiative carrier recombination processes that were controlled by the degree of non-radiative recombination with surface bound ozone [1].

A potential approach for enhancing ozone sensitivity and response/recovery rate is combined with QDs and other ozone reactive materials. Noble metal (NM) particles including $\mathrm{Pt}, \mathrm{Au}, \mathrm{Pd}$ and $\mathrm{Ag}$ are reported to have ozone reactivity such as catalytic activity and reversible adsorption-desorption [3-6]. 
Therefore, we investigated $P L$ intensity changes of NM-QD composite films and NMfree QD film by ozone gas in air.

\section{Experimental}

NM-QD composite films were prepared in the following manner. Small NM particles (NM: Pt, $\mathrm{Au}, \mathrm{Pt}-\mathrm{Pd}$ alloy $(\mathrm{Pt} / \mathrm{Pd}=85 / 15(\mathrm{w} / \mathrm{w}))$ or $\mathrm{Ag}$ were deposited on a glass plate substrate by dc sputtering method [7]. An organic solution that contained homogeneously dispersed redemitting CdSe/ZnS core-shell QDs (Invitrogen Q21721MP) was then drop-deposited on the small NM particles. For comparison, NM-free QD film on a glass plate substrate was prepared by drop-deposition of QD.

$\mathrm{PL}$ intensity and spectra of QD films with or without NM on glass substrate were measured at $25^{\circ} \mathrm{C}$ under $1 \mathrm{~atm}$ by using a spectrometer [1]. For PL measurements, the sensor film samples were excited with UV light (wavelength $=365 \mathrm{~nm}$ ). Synthetic air or air containing ozone was introduced into an experimental gas cell [1].

\section{Results and Discussion}

In air, the Pt-QD, Au-QD, Pt-Pd-QD and QDonly films emitted bright $\mathrm{PL}$, whereas the $\mathrm{PL}$ of Ag-QD film was weaker by one order of magnitude. The peak PL wavelength of these films was constant at $656 \mathrm{~nm}$, irrespective of the presence or absence of $0.5 \mathrm{ppm}$ ozone in air. The stable emission wavelength helps us to rule out any deterioration or agglomeration of QD. These NM-QD composite films and QDonly films showed reversible changes in $\mathrm{PL}$ intensity by ozone in air. However, in the AgQD film, the signal-to-noise ratio was low due to weak PL.

Figure 1 shows $P L$ intensity response of the Pt-QD, Au-QD, Pt-Pd-QD and QD-only films to $0.5 \mathrm{ppm}$ ozone in air. On exposure to $0.5 \%$ ozone in air, all films showed rapid decrease in $\mathrm{PL}$ intensity at $656 \mathrm{~nm}$. Decrease of $\mathrm{PL}$ intensity after exposure to 0.5 ppm ozone for 12 min were $9 \%, 13 \%, 11 \%$ and $6 \%$ for QD-only, Pt-QD, Au-QD and Pt-Pd-QD films, respectively. Namely, the ozone sensitivity of Pt-QD and AuQD films were higher than that of QD-only film by $43 \%$ and $27 \%$, respectively. High sensitivity to ozone suggests enhanced ozone adsorption by $\mathrm{Pt}$ and Au. Ozone sensitivity of the Pt-Pd-QD film was $32 \%$ lower than that of NM-free $Q D$ film, however, the Pt-Pd-QD film showed fast recovery of $\mathrm{PL}$ intensity in air. The low ozone sensitivity and fast $\mathrm{PL}$ recovery of the Pt-Pd-QD film are considered to reflect on the catalytic activity of Pd to ozone decomposition [5]. Fast recovery was also observed in the Au-QD film, suggesting quick ozone desorption from $\mathrm{Au}$ particles.

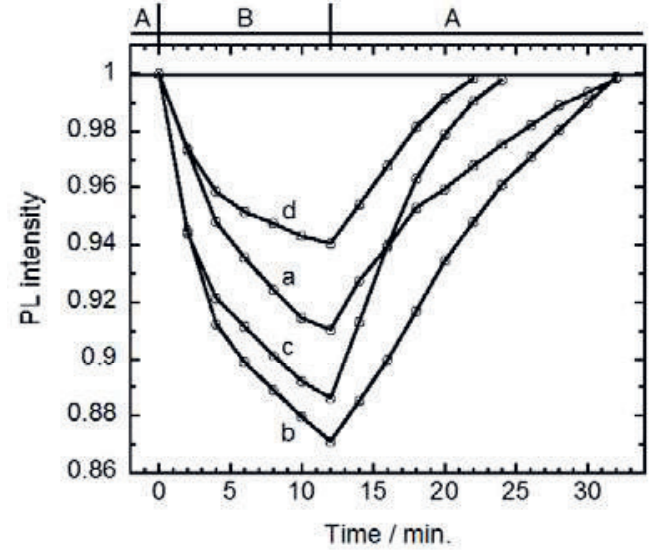

Fig. 1. Time response of $P L$ intensity at $656 \mathrm{~nm}$ for (a) a $Q D$-only, (b) a Pt-QD, (c) a Au-QD and (d) a Pt$P d-Q D$ films to $0.5 \mathrm{ppm}$ ozone in air. $A$, in air; $B$, in air containing $0.5 \mathrm{ppm}$ ozone.

\section{Acknowledgments}

This work was supported in part by JSPS KAKENHI Grant Numbers 26410201 and 17K05957 (to M. Ando and Y. Shigeri) and 17H05243 (to V. Biju).

\section{References}

[1] M. Ando, T. Kamimura, K. Uegaki, V. Biju, Y. Shigeri, Sensing of ozone based on its quenching effect on the photoluminescence of CdSe-based core-shell quantum dots, Microchim. Acta 183, 3019-3024 (2016); doi: 10.1007/s00604-0161938-9

[2] M. Ando, V. Biju, Y. Shigeri, Development of technologies for sensing ozone in ambient air, Anal. Sci. 34, 263-271 (2018); doi: 10.2116/analsci.34.263

[3] M. Petersson, D. Jonsson, H. Persson, N. Cruise, B. Andersson, Ozone promoted carbon monoxide oxidation on platinum $/ \gamma$-alumina catalyst, J. Catal. 238, 321-329 (2006); doi: 10.1016/j.jcat.2006.01.002

[4] S. D. Puckett, J. A. Heuser, J. D. Keith, W. U. Spendel, G. E. Pacey, Interaction of ozone with gold nanoparticles, Talanta 66, 1242-1246 (2005); doi: 10.1016/j.talanta.2005.01.038

[5] F. Wu, M. Wang, Y. Lu, X. Zhang, C. Yang, Catalytic removal of ozone and design of an ozone converter for the bleeding air purification of aircraft cabin, Building and Environment 115, 2533 (2017); doi: 10.1016/j.buildenv.2017.01.007

[6] T. Batakliev, G. Tyuliev, V. Georgiev, M. Anachkov, A. Eliyas, S. Rakovsky, Ozone decomposition reaction over $\alpha$-alumina-supported silver catalyst: Comparative study of catalytic surface reactivity, Ozone: Sci. \& Eng. 37, 216220 (2015); doi: 10.1080/01919512.2014.957261

[7] M. Ando, T. Kobayashi, S. lijima, M. Haruta, Optical CO sensitivity of Au-CuO composite film by use of the plasmon absorption change, Sens. Actuators B 96, 589-595 (2003); doi: 10.1016/S0925-4005(03)00645-2 